\title{
Inducing immune tolerance with dendritic cell-targeting nanomedicines
}

\author{
Anna Cifuentes-Rius ${ }^{1,4} \bowtie$, Anal Desai ${ }^{1,4}$, Daniel Yuen ${ }^{(1)}{ }^{1}$, Angus P. R. Johnston ${ }^{1}{ }^{1}$ and \\ Nicolas H. Voelcker $\mathbb{D}^{1,2,3 凶}$
}

Induced tolerogenic dendritic cells are a powerful immunotherapy for autoimmune disease that have shown promise in laboratory models of disease and early clinical trials. In contrast to conventional immunosuppressive treatments, tolerogenic immunotherapy leverages the cells and function of the immune system to quell the autoreactive lymphocytes responsible for damage and disease. The principle techniques of isolating and reprogramming dendritic cells (DCs), central to this approach, are well characterized. However, the broader application of this technology is limited by its high cost and bespoke nature. Nanomedicine offers an alternative route by performing this reprogramming process in situ. Here, we review the challenges and opportunities in using nanoparticles as a delivery mechanism to target DCs and induce immunomodulation, emphasizing their versatility. We then highlight their potential to solve critical problems in organ transplantation and increasingly prevalent autoimmune disorders such as type 1 diabetes mellitus and multiple sclerosis, where new immunotherapy approaches have begun to show promise.

D endritic cells (DCs) are a subset of antigen-presenting cells (APCs) that initiate and regulate the functions of the adaptive immune system ${ }^{1}$. Their ability to activate cytotoxic and helper $\mathrm{T}$ cells to control infections and cancer is well known, however DCs also function to restrain inappropriate autoreactive responses such as those observed in autoimmune diseases (Fig. 1). As highly phagocytic immune surveillance cells, DCs are a particularly attractive target for nanomedicines, with increasing interest in therapeutics designed to elicit tolerogenic phenotypes in these cells. Importantly, tolerogenic DCs (tolDCs) may be capable of quelling the aberrant cytotoxic and inflammatory responses in an antigen-specific manner ${ }^{2-4}$, offering a precision approach to treatment of many refractory autoimmune diseases.

An effective therapeutic designed to elicit tolDCs will need to achieve two major objectives: (1) deliver a disease appropriate 'self-antigen' to the correct population of DCs for processing and presentation, and (2) modulate or override the expression profile of these DCs to present the right signals to T cells to induce tolerogenic outcomes (Box 1). These complex prerequisites can be met with a nanomedicine approach. Nanoparticles provide a delivery vehicle capable of carrying multiple stabilized therapeutic cargos with the promising ability to generate tolDCs. Here, we examine how the optimization of the physicochemical attributes of nanoparticles can make them especially effective in designing DC-targeting nanomedicines for immune tolerance, with a focus on organ transplantation and autoimmune diseases such as type 1 diabetes mellitus (T1D) and multiple sclerosis (MS).

\section{Central and peripheral tolerance}

$T$ cells are a major mediator of damage in autoimmune disease when they aberrantly recognise and react against innocuous self-antigens in the same way as infected or cancerous cells $s^{5-7}$. In health, this is prevented by multiple tolerance mechanisms, including those found centrally in thymus and mediated peripherally by $\mathrm{DCs}^{8}$. 'Central' tolerance is enforced during $\mathrm{T}$ cell development in the thymus, where autoreactive $\mathrm{T}$ cells are removed in a process that involves thymic DCs. However, this coverage is insufficient and thus requires a second layer of regulation provided by DCs (and other APCs) in the periphery ${ }^{8}$.

DCs located throughout the body continually survey their surroundings by constantly phagocytosing nearby material ${ }^{9}$. If this sampling detects 'foreign' material such as viral RNA or bacterial membrane lipids ${ }^{10}$, a transcriptional and phenotypical transformation of the DCs termed 'maturation' occurs. Maturing DCs cease sampling their environment, present their captured antigens on major histocompatibility complex (MHC)-II, and express surface receptors that authorize the activation of antigen-specific $\mathrm{T}$ cells. These DCs then migrate to secondary lymph organs to present their collected antigens and co-stimulatory receptors to $\mathrm{T}$ cells and initiate the secondary immune response ${ }^{11}$. This combination of antigen and lymphocyte co-receptor stimulation is the basis of peripheral tolerance. If the DC-T cell encounter occurs in the absence of co-stimulatory signalling or in an environment where other signals override co-stimulation (including interaction with tolDCs), T cells can lose their effector functions and become 'anergized. These cells either differentiate into regulatory $\mathrm{T}$ cells (Tregs), which play a role in regulating or suppressing other immune cells, or are directed to undergo apoptosis (also known as 'deletion') (Box 1). This 'licensing' of T cell activity by mature DCs occurs only in the presence of foreign material, and the muting of responses in their absence restricts the activity of self-reactive $T$ cells that have escaped central tolerance mechanisms ${ }^{8}$.

\section{Generating antigen-specific tolerogenic DCs}

TolDCs for therapeutic use can be generated using methods adapted from DC vaccination. Ex vivo purified DCs are commonly cultured with tolerance-inducing pharmacological agents such as rapamycin or dexamethasone, and/or cytokines such as transforming growth factor (TGF)- $\beta$ and interleukin (IL)-10, to induce a tolerogenic phenotype ${ }^{12}$. These 'induced' tolDCs can then be reintroduced into the body. Depending on the induction method, these cells exhibit intermediate phenotypes that feature properties from both immature and activated mature DCs, including differences in migratory activity, anti-inflammatory cytokine release and the type of tolerogenic

'Monash Institute of Pharmaceutical Sciences, Monash University, Parkville Campus, Parkville, Victoria, Australia. ${ }^{2} \mathrm{CSIRO}$ Manufacturing, Bayview Avenue, Clayton, Victoria, Australia. ${ }^{3}$ Melbourne Centre for Nanofabrication, Victorian Node of the Australian National Fabrication Facility, Clayton, Victoria,

Australia. ${ }^{4}$ These authors contributed equally: Anna Cifuentes-Rius, Anal Desai. 凶e-mail: anna.cifuentesrius@monash.edu; nicolas.voelcker@monash.edu 


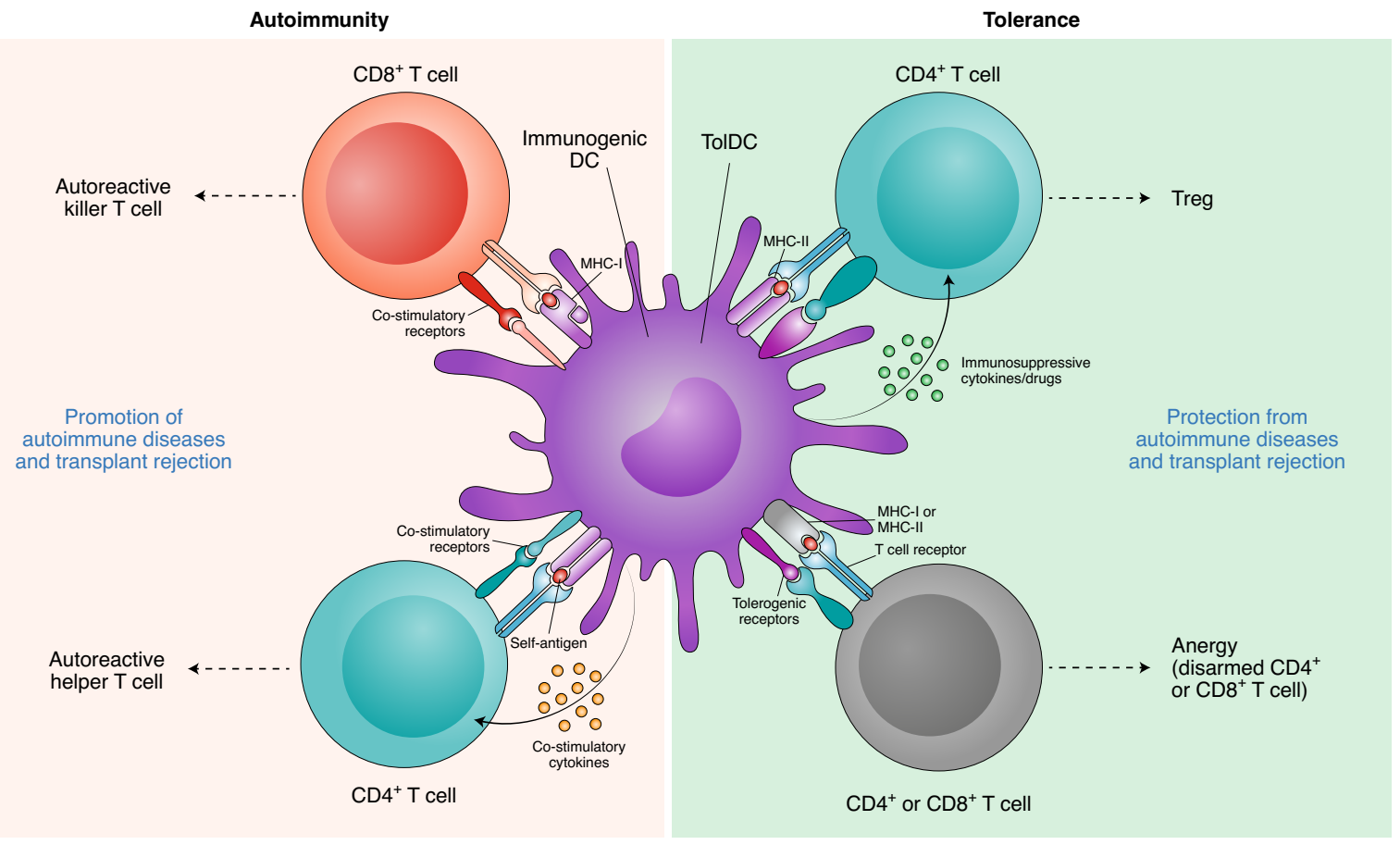

Fig. 1 | Dendritic cell function in autoimmunity versus tolerance. Dendritic cells (DCs) specifically interact with T cells through major histocompatibility complex (MHC)-I and II. However, the balance of signalling through co-receptors and cytokines determines the outcome of antigen recognition. Activated, pro-inflammatory DCs presenting self-antigen can prime auto-reactive killer clusters of differentiation (CD) $8^{+}$and $C D 4^{+}$helper T cells (left panel). Tolerogenic DCs (tolDCs) express different co-receptors and cytokines, and can induce regulatory T cells (Tregs) (upper right), as well as disarm autoreactive $\mathrm{CD} 4^{+}$and $\mathrm{CD} 8^{+} \mathrm{T}$ cells (lower right).

\section{Box 1 | Anergy, Tregs and deletion}

The therapeutic objective of tolDC induction is the quelling of cellular autoimmune responses through the induction of peripheral tolerance mechanisms. For peripheral autoreactive $\mathrm{T}$ cells exposed to tolDCs, these outcomes are one of (1) anergy, (2) conversion to a Treg phenotype or (3) T cell deletion ${ }^{2-4}$. Anergized $\mathrm{T}$ cells enter a hyporesponsive, non-dividing and functionally inactive state. For peripheral naïve $\mathrm{T}$ cells, this is thought to be non-reversible, effectively disarming the $\mathrm{T}$ cell and preventing autoimmune damage ${ }^{8}$. Autoreactive $\mathrm{T}$ cells encountering self-antigen in the presence of sufficient tolerogenic and/or environmental signals (including those from tolDCs) may instead activate the transcriptional regulator Foxp $3^{105}$, which results in functional differentiation into Tregs. Tregs can mediate further tolerogenic responses by secreting anti-inflammatory cytokines, including transforming growth factor- $\beta$ and interleukin- $10^{15}$. Lastly, autoreactive $\mathrm{T}$ cells chronically exposed to self-antigen in the absence of co-stimulation are signalled to undergo apoptosis by 'death receptors' such as cluster of differentiation (CD)95 expressed on immature DCs, simply removing them from the immune repertoire ${ }^{16}$.

response induced in $\mathrm{T}$ cells. Induced tolDCs can provide non-specific immunosuppression, however generating tolDCs to target specific autoimmune conditions requires the loading of these DCs with disease-specific autoantigens ${ }^{13}$.

In DCs, the exogenously encountered antigen is typically presented on MHC-II, with which only a cluster of differentiation (CD) $4^{+} \mathrm{T}$ cells are able to interact. For recognition by $\mathrm{CD}^{+} \mathrm{T}$ cells, exogenous antigens must be presented on MHC-I, a process that only occurs on specific DC subsets, referred to as cross-presenting DCs. Cross-presenting DCs are able to sample and load peptides onto both MHC-I and II. Cross-presentation is critical in the induction of adaptive immune responses against tumours and viruses, as well as in the induction of peripheral tolerance ${ }^{14}$. In cancer immunity, cross-presentation allows the DCs to present exogenous self-antigens to tumour-specific T cells on both MHC-I and II, priming anti-tumour responses in $\mathrm{CD}^{+}$and $\mathrm{CD} 8^{+} \mathrm{T}$ cells. Conversely, tolDCs generated from cross-presenting DCs interact with $\mathrm{T}$ cell subtypes to generate $\mathrm{CD} 4^{+}$Tregs and induce anergy in autoreactive $\mathrm{CD}^{+}$and $\mathrm{CD} 8^{+} \mathrm{T}$ cells. Importantly, cross-presenting tolDCs can also present exogenously encountered self (for example, proteins from other cells) or other innocuous antigens (for example, dietary) on MHC-I. These additional epitopes may be important for the effective control of autoimmune diseases by tolDCs in the same way multi-epitope, cross-presented antigen responses are critical in viral and cancer immunity ${ }^{14-16}$.

Despite showing promising results in clinical trials, the broader implementation of ex vivo tolDCs therapies remains challenging. Isolation, purification and culture of autologous primary DCs (or more commonly, their progenitor cells) requires extensive handling and can be prohibitively expensive, while tolDCs generated in this manner are also typically bespoke for the patient due to histocompatibility issues. An emerging alternative that overcomes both of these limitations is the in situ induction of tolDCs, where a tolerogenic payload is delivered to DCs in vivo ${ }^{17}$. Nanoparticles offer the possibility of delivering controlled amounts of self-antigen and tolerogenic agents simultaneously to individual DCs, directed by DC-specific targeting ligands. This approach would allow tolDC therapies to be accessible to a much wider range of patients and can be readily adapted for many different autoimmune diseases. Such nanoparticle-based approaches have precedents not only in the DC 
vaccination field but also in other therapeutic or clinical applications ${ }^{18,19}$, with their development offering useful insight into the challenges of developing tolDC inducing therapeutics.

\section{Tuning nanoparticle properties to maximize DC modulation}

Nanoparticles injected into the circulatory or lymphatic systems are predominantly captured by macrophages and organs in the reticuloendothelial system (for example, liver, spleen $)^{1,20}$. Nanoparticles can also be captured by precursor DCs present in the blood and immature DCs residing in peripheral tissues (for example, kidneys, skin) in a similar way to pathogens ${ }^{21}$. These discoveries sparked interest in using nanoparticles to control DC functions in immunotherapy, such as in cancer ${ }^{21}$ and viral vaccines ${ }^{22}$ and treating allergies ${ }^{23}$ and may in future provide utility in the treatment of immune phenomena such as cytokine storms, a potential sequela of viral infections such as influenza ${ }^{24}$ and SARS-CoV2 ${ }^{25}$. The use of nanoparticles to manipulate DCs offer significant advantages over conventional DC-targeting tools such as the (1) protection of antigen cargo from protease degradation, (2) co-delivery of multiple therapeutic moieties to enhance the immune response, (3) ability to control the release of the cargo and (4) reduction of off-target effects $^{21,26}$. Driven by the initial success in DC-targeting nanomedicines for cancer immunotherapy, promising strategies to modulate the immune system to treat autoimmunity are staring to be adapted and developed.

When using nanoparticles to regulate immune responses, several physicochemical parameters can be optimized to boost DC targeting and response (Fig. 2). These design factors will dictate not only how the immune system will respond but also the quality of this response $e^{3,26}$. Typically, nanoparticles are endocytosed into endo/lysosomal compartments, where the antigen is loaded onto MHC-II. Of particular interest in the induction of tolerance is delivery of the antigen to the cytoplasm of DCs using nanoparticles, ensuring additional loading of antigen onto MHC-I. Delivering antigen for presentation through both pathways is desirable, as access to the broadest range of epitopes and the ability to interact with $\mathrm{CD}^{+}$and $\mathrm{CD}^{+}$autoreactive $\mathrm{T}$ cells will likely produce the most tolerogenic impact ${ }^{14}$. There are two main strategies to enable loading onto MHC-I: (1) target nanoparticles to cross-presenting DCs, which have the native ability to take exogenous antigen and load it onto MHC-I (see 'Targeting DC subtypes' section); or (2) engineer nanoparticles to escape from the endosome and deliver their therapeutic cargo directly to the cytosol, where it can be loaded onto MHC-I $\mathrm{I}^{27,28}$

Selection of suitable material. There is a plethora of materials used in the development of DC-targeting nanomedicines for both activation and suppression of immune responses. These materials must fulfil three main requirements: they must be biocompatible, non-toxic and easy to manipulate in size, shape and surface chemistry. Promising results have been obtained with nanoformulations made of organic materials such as liposomes ${ }^{17,29,30}$, polymers including poly(lactic-co-glycolic acid) (PLGA), polylactide, poly ( $\beta$-amino esters) and polyethylene glycol (PEG) $)^{13,26,31-33}$, or inorganic materials including $\mathrm{Au}^{34,35}$ and porous $\mathrm{Si}(\mathrm{pSi})^{36,37}$. The bulk material forming the nanoparticles is important because it may itself influence the development of subsequent immune responses. Some materials have certain properties that can make them immunogenic, which make these materials ideal carriers for vaccines, but equally undesirable if the goal is to induce tolerance ${ }^{38}$.

Among all materials used for nanoparticle design, polymers are ubiquitously used in the therapeutic applications as they can be engineered in a way that enables (i) the encapsulation and therefore more effective protection of the cargo, (ii) control of the release kinetics, and (iii) the delivery of the cargo to the cytosol by providing endosomal escape mechanisms, all of which are critical to ensure effective delivery to DCs ${ }^{38}$. PLGA is the most commonly reported polymer largely because it has been approved by the FDA for a number of drug delivery applications ${ }^{19}$. The by-products generated after the degradation of PLGA can lead to the downregulation of MHC-II molecules. This potentially useful immunosuppressive effect is in contrast to other polymer-based nanoparticles that have an adjuvating effect, highlighting the importance of the properties of the polymeric carrier used to promote tolerance ${ }^{39,40}$.

Other notable materials used in the design of DC-targeting nanomedicines are $\mathrm{Au}^{41,42}$ and $\mathrm{pSi}^{43,44}$ nanomaterials, as they are biocompatible and offer the ability to easily customize the size, shape and surface chemistry in order to fine-tune cellular responses. Some studies suggest that $\mathrm{Au}$ nanoparticles do not induce DC activation despite high cellular uptake, suggesting that $\mathrm{Au}$ is minimally immunogenic ${ }^{34,35}$. pSi is a material that has been extensively used for cancer therapy given its high drug loading capacity and biodegradability ${ }^{43}$. We have demonstrated that $\mathrm{pSi}$ nanoparticles alone do not stimulate DC maturation and can therefore be another good candidate to actively deliver immunosuppressive drugs to $\mathrm{DCs}^{36,37}$.

The material forming the nanoparticle, however, is only one parameter to be considered when designing DC-targeting nanomedicines as the nanoparticle size and shape will also affect the DC-nanoparticle interaction.

Optimization of nanoparticle size and shape. Immature DCs actively sample their local environment for antigens and are able to take up a wide range of exogenous materials such as viruses, bacteria and other particulates via a variety of internalization mechanisms $s^{9,18}$. Nanoparticles with a diameter under $100 \mathrm{~nm}$ can be effectively internalized by clathrin-mediated endocytosis ${ }^{26,45}$, and DCs are also capable of internalizing larger material via phagocytosis or micropinocytosis. Nanoparticle size may not prove to be a critical parameter in terms of uptake as nanomaterials ranging from a few nanometres up to more than a micrometre have been documented to be successfully internalized by $\mathrm{DCs}^{18,46}$. However, nanoparticle size is important in dictating the transport kinetics of the antigen, affecting the ability of nanoparticles to drain to the lymph nodes and induce an adaptive immune response ${ }^{46}$. This transport to the lymph nodes has been found to be restricted to nanoparticles with a diameter under $200 \mathrm{~nm}$, allowing them to reach lymph-node-resident DCs for antigen presentation within hours of injection ${ }^{46-48}$. Nanoparticles larger than $200 \mathrm{~nm}$ either reach the lymph nodes through migratory DCs, which after internalizing nanoparticles-most likely in the blood, spleen, liver or injection site-traffic to the lymph nodes ${ }^{46,49}$, and/ or remain at the injection site ${ }^{46,50}$. As a result, direct antigen processing by DCs within the lymph nodes through small nanoparticles could lead to early $\mathrm{T}$ cell activation, which might result in an enhanced adaptive response compared to the one achieved from migratory DCs transporting larger nanoparticles ${ }^{51}$. The differences in nanoparticle biodistribution within the lymph nodes could also impact the immune response due to the nanoparticle association with different DC subpopulations by favouring for example, lymph node-resident DCs over migratory DCs.

By fine-tuning nanoparticle size, interactions with other immune cell populations could also be minimized given the differences in biodistribution and lymph drainage of nanoparticles ${ }^{52}$. Nanoparticles with virus-like sizes $(20-200 \mathrm{~nm})$ have a higher propensity to interact with $\mathrm{B}$ cells ${ }^{46}$ as free-draining nanoparticles may predominantly access B cell-resident areas within the lymph nodes. Conversely, larger nanoparticles are more likely to remain in the interstitial space before entering the lymphatics with an increased chance to be phagocytosed by barrier cells-mainly DCs and macrophages. Moreover, virus-like particles $(<200 \mathrm{~nm})$ are likely to be eliminated faster by the liver given their tendency to associate with liver sinusoidal endothelial cells, instead of $\mathrm{APCs}^{53}$. 


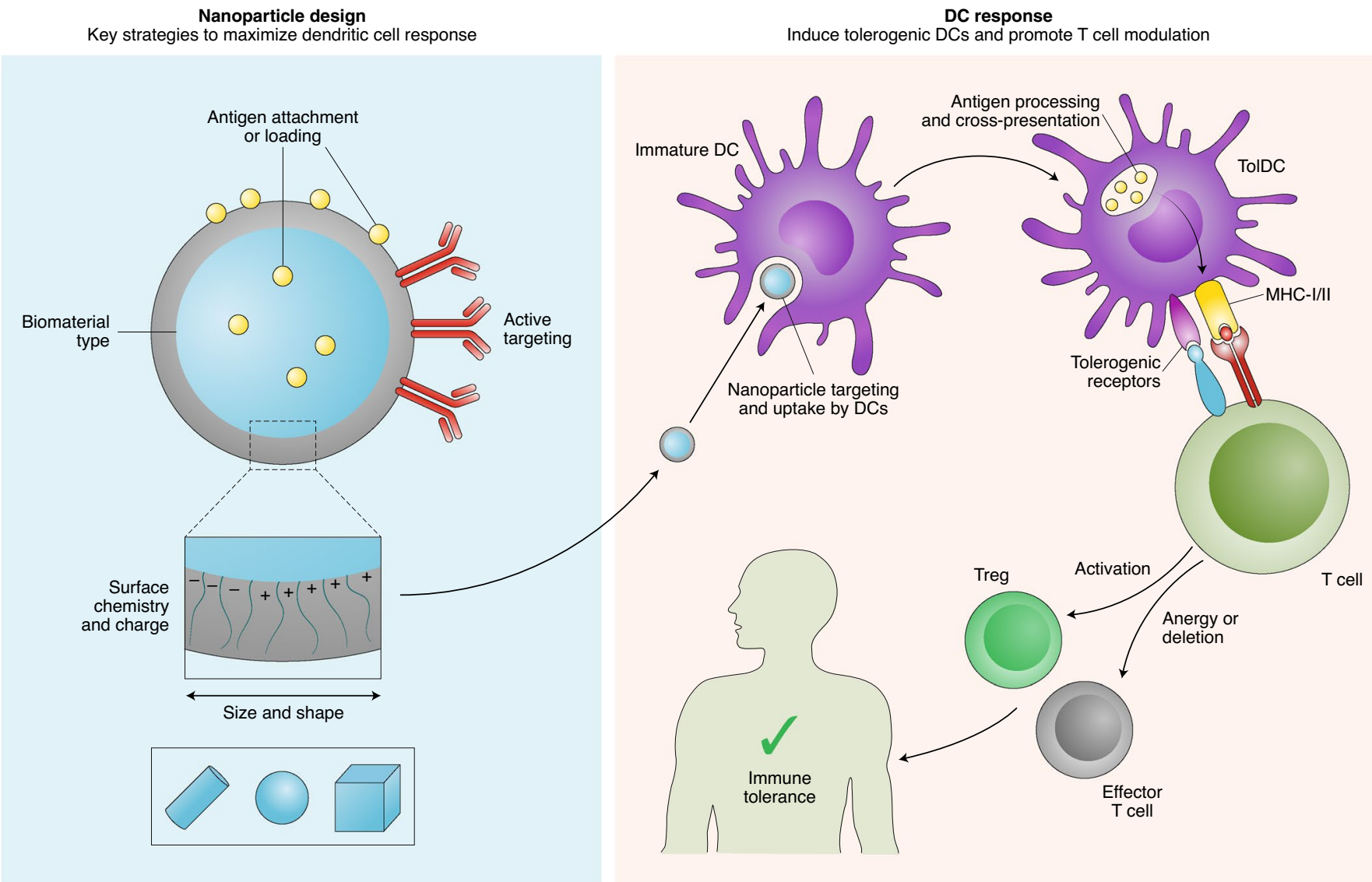

Fig. 2 | Key physicochemical characteristics of nanoparticles that will lead to maximized dendritic cell response. Nanoparticles can facilitate cytoplasmic delivery of antigen cargo, allowing access to major histocompatibility complex (MHC)-I presentation. The mechanisms of immune tolerance by dendritic cells (DCs) will provide stimulatory signals to either induce T cell anergy, maintaining T cells in their inactive state; or regulatory T cells (Tregs), which are in charge of maintaining tolerance and preventing autoimmune diseases.

Furthermore, the nanoparticle size could affect the amount of antigen loaded ${ }^{54}$, with larger nanoparticles enabling higher payload capacity that could be leveraged for optimal tolerance induction ${ }^{55}$. However, it is apparent that antigen dosage is not the only parameter leading to increased immune tolerance ${ }^{56}$.

Similarly, shape influences not only the way DCs recognise nanoparticles but also the characteristics of the subsequent immune response, and should be considered together with size ${ }^{57}$. Niikura et al., reported that spherical, rod-shaped and cubic Au nanoparticles with the same surface chemistry and protein loading generated different profiles of cytokine secretion in APCs, with differing antibody titres following vaccination in mice ${ }^{41}$. The former could not be explained solely through differential uptake, suggesting that DCs may be able to distinguish nanoparticles based on their shape. Rod-shaped nanoparticles elicited a pro-inflammatory response possibly due to lysosomal rupture-suggesting that nanorods have a greater potential as adjuvants to elicit immunity rather than tolerance. However, the influence of the nanoparticle geometry may well be size dependent, with different immune responses observed depending on the size range of the differently shaped nanoparti$\operatorname{cles}^{57}$. Nevertheless, drawing distinct conclusions between these different studies is challenging, as there are a number of differences in the experiential set-up and analysis. Multiple parameters can be normalized (for example, keeping total antigen dosage ${ }^{57}$ or total surface area $^{41}$ ) which can influence the findings.

The importance of size and shape in the design of DC-targeting nanomedicines for optimal response cannot be fully understood without looking at the nanoparticle's surface chemistry.
The nanoparticle characteristics at the surface remain a crucial player since it is the nanoparticle surface that the biological fluids, cells and tissues ultimately sense and interact with.

Modification of nanoparticle surface and charge. Different surface modifications of nanoparticles can affect their interaction and internalization into DCs. Controlling these factors is a key step in engineering nanomedicines for DC-targeting and immune modulation. The nanoparticle's surface chemistry provides properties such as hydrophilicity and charge that may facilitate or hamper the uptake and response in DCs. Highly charged nanoparticles (positive or negative) are colloidally stabilized because of electrostatic repulsion. However, in a biological setting, proteins can adsorb onto their surface-known as a protein corona-based on their surface chemistry $^{58,59}$, which can lead to aggregation, macrophage uptake and rapid clearance ${ }^{56,58}$. Moreover, studies have shown that cationic nanoparticles are more prone to be internalized by cells, including $\mathrm{DCs}^{34}$, but can also be highly immunogenic ${ }^{60}$. On the other hand, negatively charged particles have been reported to reduce acute inflammation in immune disorder ${ }^{39,61}$. Fytianos et al. studied the uptake, pro-inflammatory effects and immunological response in DCs of Au nanoparticles (15 nm) with different surface functionalities (PEG, PVA (polyvinyl alcohol) and a mixture of both) and charge (positive and negative). They demonstrated that positively charged PVA-Au nanoparticles had an increased DC uptake and a more prominent necrotic effect ${ }^{34}$. The significant decrease in cell viability of positively charged PVA-Au nanoparticles was not observed in the PEGylated counterparts, suggesting that 
cytotoxicity is depended on the combination of both the nanoparticle surface chemistry and charge. Surprisingly, among all the formulations tested, only negatively charged PEG-Au nanoparticles and positively charged PVA and PVA/PEG-Au nanoparticles promoted pro-inflammatory responses. Other studies have also shown that PVA-coated nanoparticles can induce increased co-stimulatory molecules and activation of DCs, suggesting a preference of immunity over tolerance ${ }^{62}$.

In cases where the antigen is attached to the nanoparticle surface, the number of anchoring points determines the final antigen dose administered, which has an important effect in their immunomodulation properties ${ }^{26,56}$. However, slower uptake by DCs was observed when nanoparticles were coated with the antigen on the surface as opposed to those loaded into the particle interior ${ }^{62}$. Moreover, when antigens are anchored on the nanoparticle surface, the interaction with $\mathrm{B}$ cells might be favoured given the resemblance of the antigen-coated nanoparticle to a pathogen structure ${ }^{63}$.

The ligands employed to attach antigens to nanoparticles could also be used as linkers to attach targeting moieties, such as antibodies, that are specific for DC receptors. PEG linkers are commonly used for this purpose, as their flexibility can be easily fine-tuned by manipulating the chain length to maximize cell recognition and uptake. For example, Cruz et al. showed that antigen-loaded and antibody-coated PLGA nanoparticles, PEGylated with shorter PEG chains $(2-3 \mathrm{kDa})$ exhibited not only higher DC association than those modified with larger PEG chains $(6-20 \mathrm{kDa})$ but also a more effective T cell proliferation ${ }^{64}$. Apart from the importance of the surface chemistry, this study also highlighted the role of actively targeting DCs with antibodies against specific receptor proteins.

Targeting DC subtypes. We and others have demonstrated how the incorporation of ligands targeting specific cell-surface receptors onto nanomedicine-based formulations improve internalization and localized delivery of nanotherapeutics ${ }^{44,65,66}$. Likewise, targeting specific receptors on DCs using nanomedicines not only has the potential to enhance internalization but also the ability for antigen cross-presentation, which promotes peripheral tolerance. DCs are generally broken down into four subsets based on developmental origin, surface marker and function: plasmacytoid DCs, type 1 and 2 conventional DCs, and monocyte-derived DCs. DC subsets express specific C-type lectin receptors that are in charge of antigen recognition. The specific surface markers expressed by various DC-subsets are summarized in Table $1^{67,68}$.

When nanoparticles are targeted to C-type lectin receptors, they are internalized by different cellular pathways, depending on the targeted surface receptor, and initiate a response in which DCs mature and prime the cells responsible for the adaptive response ${ }^{64,69}$. For example, polymeric particles targeted to CD11c, a generic DC marker, on cross-presenting $\mathrm{CD}^{+}{ }^{+} \mathrm{DCs}$ show efficient internalization into cells, however the same sized particles targeted to DEC205 (type 1 conventional DC specific surface marker) show limited uptake. This is despite both receptors showing rapid internalization $^{69}$. The mechanism by which particles are internalized by cells is often the subject of debate, however it has yet to be established if the mechanism of uptake plays a role in the subsequent fate and therapeutic effect of the cargo ${ }^{27}$. While there is significant potential to exploit DC-receptor mediated targeting and modulation, most nanoformulations developed to date with in vivo applications in immunotherapy do not employ active targeting strategies.

Ideally, when engineering nanomaterials to induce tolerance, the receptor used for DC active targeting should be as neutral as possible; meaning that it should mediate uptake by DCs without instructing them to prime a particular immune response ${ }^{70}$. White et al. confirmed that targeting DCs through mannosylated-liposomes increased the uptake of the nanoparticles without leading to the enhancement of $\mathrm{T}$ cell activation ${ }^{29}$. Though anti-Clec9A and
anti-DEC-205 monoclonal antibodies have also been shown to enhance DC internalization while priming Treg activation over cytotoxic T cells, they have been insufficient to promote DC activation and maturation ${ }^{71,72}$.

Dendritic cell-specific intercellular adhesion molecule-3grabbing non-integrin (DC-SIGN) is less commonly studied as a DC target than other C-type lectin receptors due to difficulties in fully recapitulating its function in currently available in vivo mod$\mathrm{els}^{73}$. Nonetheless, Stead et al. showed that pSi nanoparticles coated with anti-DC-SIGN monoclonal antibodies are capable of targeting human/non-human primate DC-SIGN in marmoset monkeys ${ }^{37}$. Cruz et al. showed that anti-DC-SIGN antibody-coated Au nanoparticles induced higher levels of cytokines ex vivo as opposed to $\mathrm{Au}$ nanoparticles without antibody or with the isotype control, suggesting that the processing of anti-DC-SIGN antibodies also induces an immune response ${ }^{64}$. Because DC-SIGN is a carbohydrate-specific receptor that recognizes glycans, other studies have used carbohydrate systems such as fucosylamide ligands to target DC-SIGN, promoting internalization without any immune responses ${ }^{35}$.

In addition to cell-surface phenotype, the anatomical location of targeted DC subpopulations must be considered. Plasmacytoid DCs reside in the bone marrow and peripheral lymphoid tissues while type 1 and 2 conventional DCs are found in peripheral tissues and eventually migrate to the lymph nodes or the spleen, respectively, upon maturation ${ }^{74}$. Thus, the localization efficacy of the DC receptor-targeted nanoparticles is highly dependent on the route of administration. To illustrate the effect of the administration route on nanoparticle internalization by certain DC subtypes, Hunter et al. reported that the same dose of disease-specific antigen-coupled nanoparticles were more effective at preventing clinical experimental autoimmune encephalomyelitis (EAE), a MS model, symptoms when administered intravenously compared to intraperitoneally; offering negligible protection following subcutaneous or oral routes ${ }^{56}$

It is therefore crucial to understand the surface markers of the DC subpopulations, their locations and the physiological cues that trigger their maturation and recruitment from the peripheral tissues to the lymphatic system to be able to then optimize the nanoparticle and its cargo for immunological response.

Selection of therapeutic cargo. Immunosuppressive drugs used in the clinic are a common choice to be loaded into and delivered by nanoparticles. Soluble administration of these drugs can induce tolDCs, but their non-specific delivery can dampen overall immunity, increasing patient susceptibility to infections and other side effects $^{75}$. Nanoparticles offer the possibility to deliver these drugs to the site of action, avoiding high systemic drug concentrations and drug exposure in healthy tissues.

Rapamycin, a macrolide used to suppress $\mathrm{T}$ cell activation and promote Treg expansion, was delivered using different nanoparticle-based carriers with the aim to induce tolDCs, especially in PLGA-based nanoparticles given their sustained drug release performance ${ }^{13,76}$. Kishimoto et al. demonstrated that rapamycin had a more durable immunological tolerance when delivered via PLGA nanoparticles than when administered on its own, likely due to a higher amount of drug internalized by $\mathrm{DCs}^{76}$.

The choice of tolerogenic agent packaged into nanoparticles is not limited to small molecule drugs. Other combinations or novel tolerogenic agents (for example, small interfering $\mathrm{RNA}^{77}$ and other oligonucleotides $^{49}$ ) can be loaded into nanoparticles and may deliver a superior tolerogenic outcome-an area well worth further investigation. In a similar manner, autoantigens can be co-delivered in nanoparticles. Antigen-specific tolDCs have been reported to induce superior tolerogenic responses compared to standard immunosuppressive treatments, with increased deletion of autoreactive $\mathrm{T}$ cells and conversion to Tregs ${ }^{13}$. The effectiveness of autoantigens 
Table 1 | Surface markers on dendritic cell subsets

\begin{tabular}{|c|c|c|c|c|}
\hline DC Subsets & Plasmacytoid DC & Type 1 conventional DC & Type 2 conventional DC & Monocyte-derived DC \\
\hline $\begin{array}{l}\text { Mouse } \\
\text { surface } \\
\text { markers }\end{array}$ & SiglecH, BST2, B22O & $\begin{array}{l}\text { Clec9A, XCR1, DEC205, CD103 } \\
\text { (heterogeneous), CD8 } \\
\text { (heterogeneous), BST2 } \\
\text { (mature cells), CD207 }\end{array}$ & $\begin{array}{l}\text { CD11b, Sirp- } \alpha, \text { BST2 (mature } \\
\text { cells), MR } \\
\text { DCIR2 (heterogeneous) }\end{array}$ & $\begin{array}{l}\text { CD11b, Sirp- } \alpha, \text { BST2 (mature cells), MR, } \\
\text { FceRI, CD64, CD14 }\end{array}$ \\
\hline $\begin{array}{l}\text { Human } \\
\text { surface } \\
\text { markers }\end{array}$ & CD123, BDCA-2, BDCA-4 & XCR-1, Clec9A, BDCA-3 & BDCA-1, CD11b, Sirp- $\alpha$ & $\begin{array}{l}\text { BDCA-1, CD11b, Sirp- } \alpha, \text { CD1a, MR, } \\
\text { DC-SIGN (heterogeneous), FceRI, } \\
\text { CD64, CD14 }\end{array}$ \\
\hline Location & $\begin{array}{l}\text { Bone marrow and } \\
\text { peripheral lymphoid } \\
\text { tissues }\end{array}$ & $\begin{array}{l}\text { Lymph nodes and peripheral } \\
\text { tissues }\end{array}$ & Spleen and peripheral tissues & $\begin{array}{l}\text { Recruited from the bone marrow to the } \\
\text { lymphoid and peripheral tissues in an } \\
\text { inflammatory setting }\end{array}$ \\
\hline Function & $\begin{array}{l}\text { IFN-I and III production, } \\
\text { priming of NK cells }\end{array}$ & $\begin{array}{l}\text { Antigen uptake and presentation, } \\
\text { priming } C D 8^{+} T \text { cells }\end{array}$ & $\begin{array}{l}\text { Antigen uptake and } \\
\text { presentation, priming } \mathrm{CD} 4^{+} \\
\text {T cells }\end{array}$ & $\begin{array}{l}\text { Induction of pro-inflammatory cytokines } \\
\text { during inflammation }\end{array}$ \\
\hline
\end{tabular}

SiglecH, sialic acid binding Ig-like lectin $\mathrm{H} ; \mathrm{BST} 2$, bone marrow stromal cell antigen 2; CD, cluster of differentiation; PDCA-1, plasmacytoid dendritic cell antigen-1; Clec9A, C-type lectin domain family 9-member A; XCR-1, chemokine XC receptor-1; Sirp- $\alpha$, signal regulatory protein alpha; MR, mannose receptor; DCIR2, dendritic cell immunoreceptor 2; DC-SIGN, dendritic cell-specific intercellular adhesion molecule-3-grabbing non-integrin; FceRI, the high affinity receptor for immunoglobulin E. Markers designated as 'heterogeneous' are tissue-specific.

has been demonstrated using the widely used chicken ovalbumin model $^{13,37}$, as well as in disease models such as proteolipid protein in $\mathrm{MS}^{56}$. Importantly, several studies have reported immunogenic responses when the antigen is delivered by nanoparticles in the absence of immunomodulatory agents ${ }^{32,54,78}$. Combined delivery of tolerogenic agents and autoantigen within a single nanoparticle is likely critical to ensure that an individual DC receives all the necessary signals to induce an optimal tolerogenic response and stimulate Treg proliferation ${ }^{32,37}$. Co-delivery will be particularly important where an inflammatory environment already exists and can override the induced tolerogenic respons $\mathrm{e}^{32}$. Interestingly, others have reported tolerogenic activity using nanoparticles coupled with antigen alone $\mathrm{e}^{30,32}$. A possible explanation could be that a combination of large $(500-1000 \mathrm{~nm})$ and highly negatively charged $(-10$ to $-60 \mathrm{mV})$ nanoparticles resemble apoptotic bodies which may inhibit immunostimulatory activation of DCs and thus induce tolerance ${ }^{54,78,79}$. These discrepancies highlight the importance of the interplay between different physicochemical characteristics of nanoparticles in their interaction with DCs.

Engman et al., on the other hand, demonstrated that the delivery of an antigen was not a prerequisite to generate Tregs by delivering stimulatory protein-targeted antisense oligonucleotides ${ }^{49}$. This strategy was able to successfully promote tolDCs and in vivo generation of antigen-specific Tregs.

\section{Applying nanoparticles in transplantation tolerance}

Allograft transplantation is often the only available alternative for patients who face end-stage organ disease, with the rejection of donor organs by the host remaining a major clinical challenge ${ }^{80}$. Rejection is initiated when the donor antigens in the transplant are recognized by the immune system of the recipient. Immunosuppressants can reduce chronic graft rejection, but their prolonged administration leaves the patient vulnerable to opportunistic infections and cancer ${ }^{81}$. DCs are critical players in the rejection or survival of transplants given their capacity to internalize and cross-present alloantigens and thus are considered a logical target to modulate immunity after transplantation ${ }^{82}$. As represented in Fig. 3, several nanoparticle-based formulations have shown promise in fighting transplant rejection. The delivery of rapamycin via intradermally injected PEGylated micelles has, for example, shown significantly prolonged survival of skin allografts ${ }^{31}$. This study suggests that nanoparticles could potentially prevent some of the long-term implications related to the non-specific delivery of immunosuppressants.
However, the 'Holy Grail' of transplantation has been the induction of donor-specific immune tolerance without generalized immunosuppression ${ }^{83}$. This approach induces immunological tolerance against specific antigens while maintaining an otherwise functional immune system. Nanoparticles can be used to induce donor-specific tolerance that is long-lasting. For instance, Bryant et al. investigated the ability of PLGA-based nanoparticles to deliver allogeneic donor antigens in a mismatched islet cell transplantation model $^{84}$. They demonstrated how nanoparticles loaded with donor antigens offer long-term protection of transplanted islet allografts when combined with low doses of rapamycin. In a follow-up study, they observed how intravenously administered nanoparticles loaded with a tissue-specific antigen induced donor-specific tolerance in a mismatched bone marrow transplant model ${ }^{85}$.

Despite being still relatively underexplored, using nanoparticles as a platform to produce tolDCs in organ transplantation appears to be a promising strategy as a co-therapy for cell and tissue transplantation by extending the durability of cell therapies without the need of high doses of systemic immunosuppressants.

\section{Potential for nanoparticles in immunomodulation of autoimmune disorders}

Autoimmune disorders such as T1D, MS, rheumatoid arthritis and inflammatory bowel disease result from failure to sustain tolerance to self-antigens. We have discussed how tolerogenic nanoparticles can induce tolDCs, which can be leveraged to treat autoimmune diseases (Fig. 3). DC-targeting nanoparticles have shown potential in treating T1D and MS and could be further employed to improvise already proven cargos and targets.

Type 1 diabetes mellitus. T1D is an autoimmune disorder characterized by an immune response against $\beta$ cell antigens, in which the host immune system induces a targeted $\mathrm{CD} 4^{+}$and $\mathrm{CD} 8^{+} \mathrm{T}$ cell response against insulin-producing $\beta$ cells in the pancreas ${ }^{86}$. The resulting dysregulation of insulin secretion, maintenance and eventual loss of controlled blood glucose variability facilitates the onset of severe complications such as diabetic retinopathy, neuropathy, cardiovascular disease and renal failure. The current treatment of administering long-term pharmacologic insulin supplementation prolongs the onset of diabetes-associated pathophysiology but does not address the rapid fluctuations in blood glucose levels, nor does it address the underlying autoimmune pathophysiology of the disease. Concurrently, broad immunosuppression or immunoablation followed by bone marrow or islet 


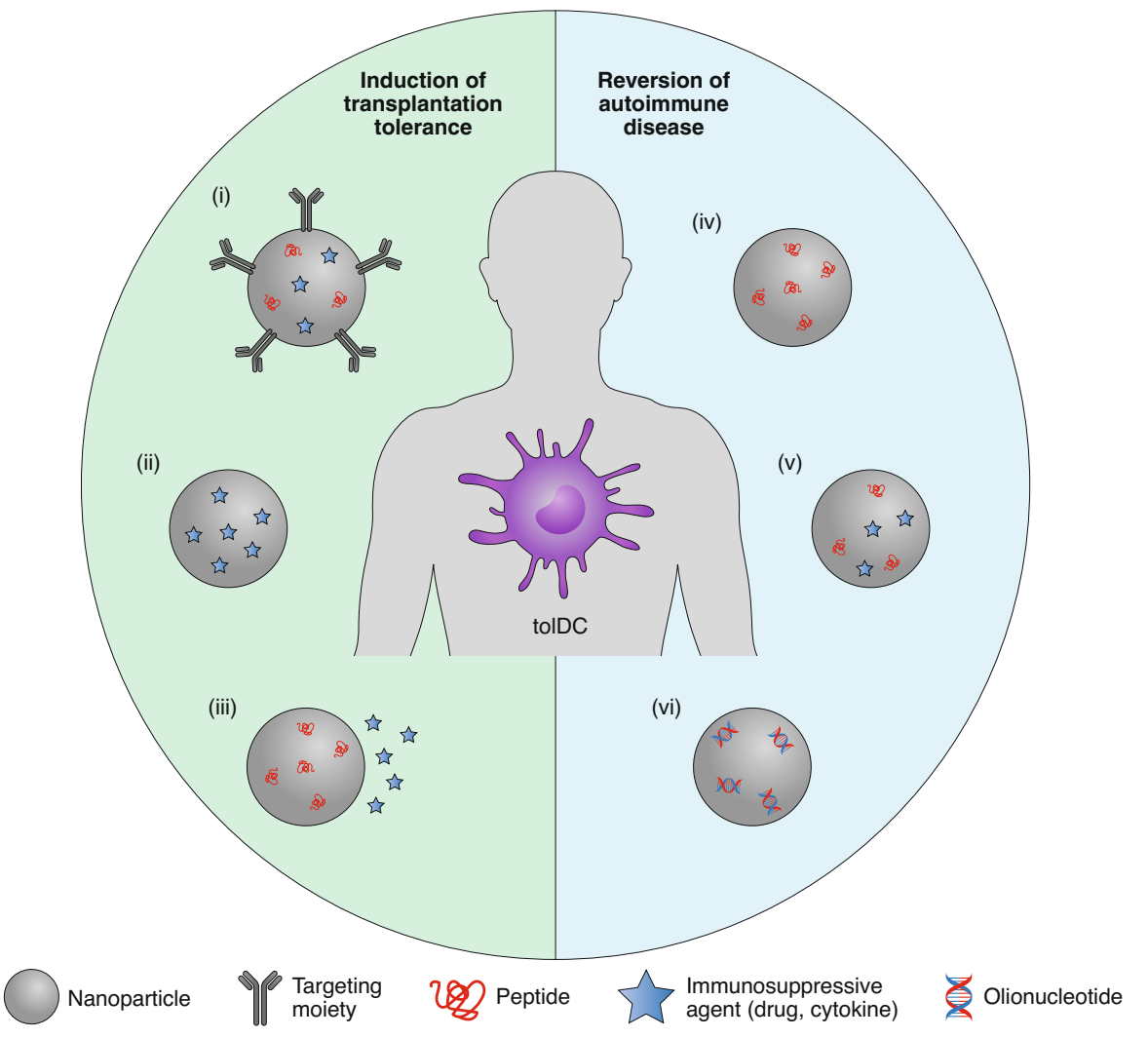

\begin{tabular}{|c|c|c|c|c|c|}
\hline & Material & $\begin{array}{c}\text { Nanoparticle } \\
\text { size }(\mathrm{nm})\end{array}$ & Therapeutic cargo & Disease & Ref. \\
\hline (i) & $\mathrm{pSi}$ & 200 & $\mathrm{OVA}_{323-339}$ and/or rapamycin & Transplantation & 37 \\
\hline (ii) & PEG-bl-PPS & 50 & $\begin{array}{l}\text { Dexamethasone, mometasone furoate, } \\
\text { rapamycin and tacrolimus }\end{array}$ & Transplantation & 31 \\
\hline (iii) & PLGA & 450 & Allogenic donor antigen and free rapamycin & Transplantation & 84 \\
\hline (iii) & PLGA & 450 & $\mathrm{OVA}_{323-339}$ and/or rapamycin & Transplantation & 85 \\
\hline (iv) & PS-Liposomes & 1,000 & Insulin A and B peptides & T1D & 30 \\
\hline (iv) & PLGA and polylactide & 500 & $\mathrm{OVA}_{323-339}$ and PLP ${ }_{139-151}$ & MS & 39 \\
\hline (iv) & PLGA and polylactide & 500 & $\mathrm{OVA}_{323-339}$ and $\mathrm{PLP}_{139-151}$ & MS & $30,39,56,98$ \\
\hline (v) & PLGA & N/A & $\mathrm{PLP}_{139-151}$ and rapamycin & MS & 32 \\
\hline (v) & $\mathrm{Au}$ & 60 & $\mathrm{MOG}_{35-55}$ and ITE & MS & 78 \\
\hline (v) & $\mathrm{Au}$ & 60 & ITE and proinsulin & T1D & 92 \\
\hline (v) & PLGA & 1,000 and 30,000 & $\begin{array}{c}\text { Vitamin D3 and insulin B9-23 }(1 \mu \mathrm{m}) \text { and } \\
\text { GM-CSF and TGF- } \beta 1(30 \mu \mathrm{m})\end{array}$ & T1D & 50 \\
\hline (v) & PLGA & 200 & $\mathrm{MOG}_{35-55}$ and IL-10 & MS & 97 \\
\hline (vi) & PLGA & $1,000-1,800$ & CD40, CD80 and CD86 oligonucleotides & T1D & 49 \\
\hline
\end{tabular}

Fig. 3 | Summary of nanoformulations developed to promote tolerogenic dendritic cells through dendritic cell-T cell interactions to either combat transplantation rejection (green) or autoimmune diseases (blue). Nanomedicines enabling: (i) active targeted delivery of antigens and immunosuppressive agents (this study does not use a transplantation model per se, but proves that nanoparticles actively target dendritic cells (DCs) in vivo inducing

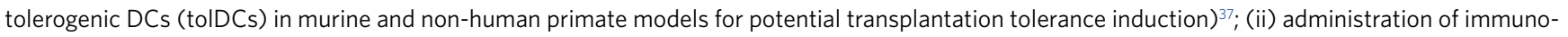
suppressive drugs loaded into nanoparticle formulations ${ }^{31}$; (iii) delivery of antigen-loaded nanoparticles together with free immunomodulatory agents ${ }^{84,85}$; (iv) administration of antigen-nanoparticle conjugates ${ }^{30,39,56,98 ;}$ ( V) co-delivery of immunosuppressive drugs loaded into nanoparticles ${ }^{32,50,78,92,97}$; and (vi) delivery of oligonucleotides ${ }^{49}$. OVA, chicken egg albumin; MOG, myelin oligodendrocyte glycoprotein; ITE, 2-(1' $\mathrm{H}$-indole-3'-carbonyl)-thiazole4-carboxylic acid methyl ester; GM-CSF, granulocyte-macrophage colony-stimulating factor.

transplantation has been demonstrated to be effective in animal models, but in the clinical setting, patients continue to encounter some level of tissue rejection in addition to other limitations such as high cost and availability of donor islet cells ${ }^{87}$. TolDCs represent a promising approach for the treatment of T1D where long-term, antigen-specific immune tolerance is achieved by 
inducing $\mathrm{T}$ cell anergy or selective apoptosis and simultaneously stimulating subpopulations of Tregs and regulatory B cells, which in turn transfer the immunoregulatory properties to naïve $\mathrm{T}$ cells in a feedback loop ${ }^{88}$. Like Tregs, regulatory B cells participate in the suppression of immune responses primarily via the production of IL-10, TGF- $\beta$ and IL- $35^{89}$. Deficits in pancreatic Foxp $3^{+}$Tregs, in both number and functionality, have been described in T1D patients $^{88,89}$. Nanoparticles can prove to be vital in such instances where it is essential to co-deliver the disease-specific autoantigen and other tolerogenic agents to modify a tissue microenvironment. Lewis et al. exploited the immunosuppressive capabilities of PLGA and generated a system to co-deliver protolerogenic agents via large, nonphagocytosable particles that release DC chemokine in the extracellular space and insulin using the smaller, phagocytosable particles that recruit and tolerize DCs. With this approach, they not only observed an increase in Tregs but also an upregulation of the protein, programmed cell death protein-1, a marker for self-tolerance, on $\mathrm{CD}^{+}$and $\mathrm{CD}^{+} \mathrm{T}$ cells and reverse hyperglycemia ${ }^{50}$. Similarly, a diverse range of other immunosuppressive and tolDC inducing agents have also been reported to induce a tolerogenic phenotype, some of which are already being applied in nanoparticle-based combination therapies ${ }^{90-92}$. IL-2, IL-3, IL-10, IL-12, IL-21, type I interferons, tumour necrosis factor alpha, adenosine deaminase, protein tyrosine phosphatase non-receptor type 22 and B lymphocyte-induced maturation protein-1 have all been closely associated with DC phenotype in non-obese diabetic mice ${ }^{86,91}$ but their induction or suppression, as a part of combination therapy, remains to be tested.

Another major advantage of using nanoparticles in T1D is its ability to target DC surface markers. Antibody-based approaches have been successful in inducing tolerogenic responses in preclinical models, but have not progressed due to unfavourable pharmacokinetics and interactions with the immune system ${ }^{90,91}$. Surprisingly, nanoparticles functionalized with antibodies against DC-surface markers have rarely been used for this application and thus provide an avenue for well-established nanoparticle platforms to further improve their effectiveness in treating T1D. Interestingly, Engman et al. demonstrated that untargeted tolerogenic nanoparticles could be delivered to the pancreas by injecting them into the associated draining lymph nodes. Resident DCs in these sites obtained autoantigens in situ, generating antigen-specific Foxp $3^{+}$Treg with nanoparticles providing only tolerogenic stimuli (knockdown of co-stimulatory molecules) ${ }^{49}$. This non-targeted approach exploits the facile lymphoid accessibility of the pancreas, but for more anatomically complex or diffuse autoimmune conditions such an approach may not be effective. A broadly successful nanoparticle-based therapeutic is likely to comprise a DC subset targeting delivery of the disease antigen in parallel with one or more tolerogenic agents.

Multiple sclerosis. MS is an autoimmune disease characterized by chronic inflammation of neuronal roots that causes demyelination in the central nervous system resulting in motor, sensory and cognitive deficits ${ }^{93}$. Though the precise cellular mechanisms involved in the immune deregulation are not known, in MS, the DC-activated $\mathrm{CD}^{+} \mathrm{T}$ cells in the peripheral draining lymph nodes cross the blood brain barrier and infiltrate the central nervous system where they are restimulated by local DCs resulting in inflammation of the myelin and axonal cells ${ }^{93}$. Ablation of DCs in the EAE model did not alter disease incidence but exhibited amelioration of disease severity, indicating that DCs play a role in priming pathogenic $\mathrm{T}$ cells or generating the inflammatory milieu. Subsequently, Treg induction and stimulation of $\mathrm{CD}_{11 \mathrm{~b}^{+}}$and CD $11 \mathrm{c}^{+}$DCs have been directly associated with a tolerogenic phenotype in EAE mice ${ }^{94,95}$. Conventional therapies for MS include oral or intravenous immunomodulatory/immunosuppressive treatments supplemented with cytokine or monoclonal antibodies. These approaches not only fail to address MS symptoms rather than the immune cell dysregulation, but have also been reported to have significant side effects after prolonged use ${ }^{96}$; factors that emerging antigen-specific immunotherapies have successfully overcome via a targeted suppression of the immune responses ${ }^{32,78,97}$. Nanoparticle properties such as bulk biomaterial ${ }^{39}$, antigen coupling $^{56,98}$ and co-delivery of tolerogenic agents (Fig. 3) have been studied to improve the disease antigen administration. For example, Maldonado et al. (delivering MS autoantigen and rapamycin), and Capellano et al. (delivering disease antigen with IL-10, a CD4 ${ }^{+}$ $\mathrm{T}$ cell tolerance inducing cytokine), showed that administration of PLGA nanoparticles with combinatory cargo localized to DCs in draining lymph nodes and improved clinical scores in EAE mice ${ }^{32,97}$. Similarly, nanoparticles can prove crucial in increasing the effectiveness of tolerogenic response where immunosuppressive and immunoprotective agents (for example, interferon $\beta$, IL-17, IL-23, vitamin D3) have already shown a strong association with Treg induction and lower MS incident ${ }^{6,99-101}$. Lastly, just as in T1D, there have been attempts made to deliver the autoantigens with antibodies targeting DEC-205 ${ }^{102,103}$, dendritic cell immunoreceptor $2^{103}$ and sialic acid binding Ig-like lectin $\mathrm{H}^{104}$ receptors, which have achieved some immunological tolerance. The versatility of nanoparticles can be greatly expanded by conjugating these or other antibodies with DC surface markers in addition to the optimized cargo.

Nanoparticles conjugated with autoantigens and/or in combination with tolerogenic agents have also been implemented in other autoimmune disease models such as inflammatory bowel disease and rheumatoid arthritis and the suggestions to improvise the nanoparticle design can be applied to them ${ }^{61,76}$.

\section{Conclusions and outlook}

The use of nanoparticles to target DCs in vivo represents a promising strategy to modulate immune responses in patients with autoimmune disorders or following organ transplantation. Nanoparticle properties can be fine-tuned to deliver multiple therapeutics in order to maximize their DC-targeting, internalization and cross-presentation while reducing unwanted side effects. We have reviewed different possible approaches involving nanoparticles that can facilitate the induction of tolDCs and have assembled a set of fundamental design rules that could lead to better therapeutic outcomes. Surprisingly, there is a conspicuous lack of studies focusing on DC-targeting nanoparticles for autoimmunity, making this approach a technology still in its infancy. Despite the substantial potential, the complexity associated with the immune system and the multiple mechanisms of action has probably limited the development of this approach. The durability of the immune tolerance induced by nanoparticles has not yet been thoroughly evaluated, and a sustained response may possibly require multiple rounds of nanoparticle administration. Study of the long-term implications of tolerogenic nanoparticles, in terms of safety and side effects, is also required. Nanoparticles with complex compositions may experience difficulties in manufacture and regulatory approval. These are all important challenges that need to be addressed before nanoparticle-mediated tolerance induction is ready for clinical evaluation.

To control autoimmunity and advance the field, next-generation nanoparticles will require a convergence between immunologists, cell biologists and nanotechnologists to identify the best nanoparticle design; based on the optimal choice of therapeutics and route of administration, animal model and long-term validation techniques.

Received: 5 July 2020; Accepted: 29 October 2020;

Published online: 21 December 2020 


\section{References}

1. Hasegawa, H. \& Matsumoto, T. Mechanisms of tolerance induction by dendritic cells in vivo. Front. Immunol. 9, 350-350 (2018).

2. Obregon, C., Kumar, R., Pascual, M. A., Vassalli, G. \& Golshayan, D. Update on dendritic cell-induced immunological and clinical tolerance. Front. Immunol. 8, 1514 (2017).

3. Audiger, C., Rahman, M. J., Yun, T. J., Tarbell, K. V. \& Lesage, S. The importance of dendritic cells in maintaining immune tolerance. J. Immunol. 198, 2223 (2017)

4. Manicassamy, S. \& Pulendran, B. Dendritic cell control of tolerogenic responses. Immunol. Rev. 241, 206-227 (2011).

5. Firestein, G. S. Evolving concepts of rheumatoid arthritis. Nature 423, 356-361 (2003).

6. Frohman, E. M., Racke, M. K. \& Raine, C. S. Multiple sclerosis-the plaque and its pathogenesis. N. Engl. J. Med. 354, 942-955 (2006).

7. Zamvil, S. et al. T-cell clones specific for myelin basic protein induce chronic relapsing paralysis and demyelination. Nature 317, 355-358 (1985)

8. Xing, Y. \& Hogquist, K. A. T-cell tolerance: central and peripheral. Cold Spring Harb. Perspect. Biol. https://doi.org/10.1101/cshperspect.a006957 (2012).

9. Ezekowitz, R. A. B. \& Hoffmann, J. A. Innate immunity. Curr. Opin. Immunol. 8, 1-2 (1996).

10. Beutler, B. Microbe sensing, positive feedback loops, and the pathogenesis of inflammatory diseases. Immunol. Rev. 227, 248-263 (2009).

11. Dalod, M., Chelbi, R., Malissen, B. \& Lawrence, T. Dendritic cell maturation: functional specialization through signaling specificity and transcriptional programming. EMBO J. 33, 1104-1116 (2014).

12. Boks, M. A. et al. IL-10-generated tolerogenic dendritic cells are optimal for functional regulatory $\mathrm{T}$ cell induction-a comparative study of human clinical-applicable DC. Clin. Immunol. 142, 332-342 (2012).

13. Stabler, C. L., Li, Y., Stewart, J. M. \& Keselowsky, B. G. Engineering immunomodulatory biomaterials for type 1 diabetes. Nat. Rev. Mater. 4, 429-450 (2019).

14. Heath, W. R. \& Carbone, F. R. Cross-presentation in viral immunity and self-tolerance. Nat. Rev. Immunol. 1, 126-134 (2001).

15. Schwartz, R. H. T cell anergy. Annu. Rev. Immunol. 21, 305-334 (2003).

16. Dominguez-Villar, M. \& Hafler, D. A. Regulatory T cells in autoimmune disease. Nat. Immunol. 19, 665-673 (2018).

17. Capini, C. et al. Antigen-specific suppression of inflammatory arthritis using liposomes. J. Immunol. 182, 3556-3565 (2009).

18. Bachmann, M. F. \& Jennings, G. T. Vaccine delivery: a matter of size, geometry, kinetics and molecular patterns. Nat. Rev. Immunol. 10, 787-796 (2010).

19. Bobo, D., Robinson, K. J., Islam, J., Thurecht, K. J. \& Corrie, S. R Nanoparticle-based medicines: a review of FDA-approved materials and clinical trials to date. Pharm. Res. 33, 2373-2387 (2016).

20. Morelli, A. E. \& Thomson, A. W. Tolerogenic dendritic cells and the quest for transplant tolerance. Nat. Rev. Immunol. 7, 610-621 (2007).

21. Jia, J. et al. Interactions between nanoparticles and dendritic cells: from the perspective of cancer immunotherapy. Front. Oncol. 8, 404-404 (2018).

22. Sulczewski, F. B., Liszbinski, R. B., Romao, P. R. T. \& Rodrigues Junior, L. C. Nanoparticle vaccines against viral infections. Arch. Virol. 163, 2313-2325 (2018).

23. Al-Halifa, S., Gauthier, L., Arpin, D., Bourgault, S. \& Archambault, D. Nanoparticle-based vaccines against respiratory viruses. Front. Immunol. 10 22 (2019).

24. Liu, Q., Zhou, Y. H. \& Yang, Z. Q. The cytokine storm of severe influenza and development of immunomodulatory therapy. Cell. Mol. Immunol. 13, 3-10 (2016).

25. Rodriguez, Y. et al. Autoinflammatory and autoimmune conditions at the crossroad of COVID-19. J. Autoimmun. 114, 102506 (2016).

26. Getts, D. R., Shea, L. D., Miller, S. D. \& King, N. J. C. Harnessing nanoparticles for immune modulation. Trends Immunol. 36, 419-427 (2015)

27. Selby, L. I., Cortez-Jugo, C. M., Such, G. K. \& Johnston, A. P. R. Nanoescapology: progress toward understanding the endosomal escape of polymeric nanoparticles. Wiley Interdiscip. Rev. Nanomed. Nanobiotechnol. 9, e1452 (2017).

28. Smith, S. A., Selby, L. I., Johnston, A. P. R. \& Such, G. K. The endosomal escape of nanoparticles: toward more efficient cellular delivery. Bioconjug. Chem. 30, 263-272 (2019).

29. White, K. L., Rades, T., Furneaux, R. H., Tyler, P. C. \& Hook, S Mannosylated liposomes as antigen delivery vehicles for targeting to dendritic cells. J. Pharm. Pharmacol. 58, 729-737 (2006).

30. Pujol-Autonell, I. et al. Use of autoantigen-loaded phosphatidylserineliposomes to arrest autoimmunity in type 1 diabetes. PLoS One 10, e0127057 (2015).

31. Dane, K. Y. et al. Nano-sized drug-loaded micelles deliver payload to lymph node immune cells and prolong allograft survival. J. Control. Release 156, 154-160 (2011).
32. Maldonado, R. A. et al. Polymeric synthetic nanoparticles for the induction of antigen-specific immunological tolerance. Proc. Natl Acad. Sci. USA 112, E156 (2015).

33. Fornaguera, C. et al. mRNA delivery system for targeting antigen-presenting cells in vivo. Adv. Healthc. Mater. 7, 1800335 (2018).

34. Fytianos, K. et al. Uptake efficiency of surface modified gold nanoparticles does not correlate with functional changes and cytokine secretion in human dendritic cells in vitro. Nanomedicine 11, 633-644 (2015).

35. Arosio, D. et al. Effective targeting of DC-SIGN by $\alpha$-fucosylamide functionalized gold nanoparticles. Bioconjug. Chem. 25, 2244-2251 (2014)

36. Stead, S. O. et al. Manipulating human dendritic cell phenotype and function with targeted porous silicon nanoparticles. Biomaterials 155, 92-102 (2018)

37. Stead, S. O. et al. Murine and non-human primate dendritic cell targeting nanoparticles for in vivo generation of regulatory T-Cells. ACS Nano 12, 6637-6647 (2018).

38. Urbanavicius, D., Alvarez, T., Such, G. K., Johnston, A. P. R. \& Mintern, J. D. The potential of nanoparticle vaccines as a treatment for cancer. Mol. Immunol. 98, 2-7 (2018).

39. Saito, E. et al. Designing drug-free biodegradable nanoparticles to modulate inflammatory monocytes and neutrophils for ameliorating inflammation. J. Control. Release 300, 185-196 (2019).

40. Allen, R. P., Bolandparvaz, A., Ma, J. A., Manickam, V. A. \& Lewis, J. S Latent, immunosuppressive nature of poly(lactic-co-glycolic acid) microparticles. ACS Biomater. Sci. Eng. 4, 900-918 (2018).

41. Niikura, K. et al. Gold nanoparticles as a vaccine platform: influence of size and shape on immunological responses in vitro and in vivo. ACS Nano 7, 3926-3938 (2013)

42. Sun, X. et al. Tracking stem cells and macrophages with gold and iron oxide nanoparticles - the choice of the best suited particles. Appl. Mater. Today 15, 267-279 (2019).

43. Tieu, T., Alba, M., Elnathan, R., Cifuentes-Rius, A. \& Voelcker, N. H. Advances in porous silicon-based nanomaterials for diagnostic and therapeutic applications. Adv. Ther. 2, 1800095 (2019).

44. Kim, B. et al. Securing the payload, finding the cell, and avoiding the endosome: peptide-targeted, fusogenic porous silicon nanoparticles for delivery of siRNA. Adv. Mater. 31, 1902952 (2019).

45. Behzadi, S. et al. Cellular uptake of nanoparticles: journey inside the cell. Chem. Soc. Rev. 46, 4218-4244 (2017).

46. Manolova, V. et al. Nanoparticles target distinct dendritic cell populations according to their size. Eur. J. Immunol. 38, 1404-1413 (2008).

47. Rincon-Restrepo, M. et al. Vaccine nanocarriers: Coupling intracellular pathways and cellular biodistribution to control CD4 vs CD8 T cell responses. Biomaterials 132, 48-58 (2017).

48. Shima, F., Uto, T., Akagi, T., Baba, M. \& Akashi, M. Size effect of amphiphilic poly(gamma-glutamic acid) nanoparticles on cellular uptake and maturation of dendritic cells in vivo. Acta Biomater. 9, 8894-8901 (2013).

49. Engman, C. et al. Generation of antigen-specific Foxp3+ regulatory T-cells in vivo following administration of diabetes-reversing tolerogenic microspheres does not require provision of antigen in the formulation. Clin. Immunol. 160, 103-123 (2015).

50. Lewis, J. S. et al. Dual-sized microparticle system for generating suppressive dendritic cells prevents and reverses type 1 diabetes in the nonobese diabetic mouse model. ACS Biomater. Sci. Eng. 5, 2631-2646 (2019).

51. Gerner, M. Y., Torabi-Parizi, P. \& Germain, R. N. Strategically localized dendritic cells promote rapid $\mathrm{T}$ cell responses to lymph-borne particulate antigens. Immunity 42, 172-185 (2015).

52. Porter, C. J. H. \& Trevaskis, N. L. Nat. Nanotechnol. 15, 423-425 (2020)

53. Mates, J. M. et al. Mouse liver sinusoidal endothelium eliminates HIV-like particles from blood at a rate of 100 million per minute by a second-order kinetic process. Front. Immunol. https://doi.org/10.3389/fimmu.2017.00035 (2017).

54. Kim, S. H. et al. Induction of antigen-specific immune tolerance using biodegradable nanoparticles containing antigen and dexamethasone. Int. J. Nanomedicine 14, 5229-5242 (2019).

55. Kuo, R., Saito, E., Miller, S. D. \& Shea, L. D. Peptide-conjugated nanoparticles reduce positive co-stimulatory expression and $\mathrm{T}$ cell activity to induce tolerance. Mol. Ther. 25, 1676-1685 (2017).

56. Hunter, Z. et al. A biodegradable nanoparticle platform for the induction of antigen-specific immune tolerance for treatment of autoimmune disease. ACS Nano 8, 2148-2160 (2014).

57. Kumar, S., Anselmo, A. C., Banerjee, A., Zakrewsky, M. \& Mitragotri, S. Shape and size-dependent immune response to antigen-carrying nanoparticles. J. Control. Release 220, 141-148 (2015).

58. Cifuentes-Rius, A., de Puig, H., Kah, J. C. Y., Borros, S. \& Hamad-Schifferli, K. Optimizing the properties of the protein corona surrounding nanoparticles for tuning payload release. ACS Nano 7, 10066-10074 (2013). 
59. Johnston, B. D. et al. Colloidal stability and surface chemistry are key factors for the composition of the protein corona of inorganic gold nanoparticles. Adv. Funct. Mater. 27, 1701956 (2017).

60. Yan, W., Chen, W. \& Huang, L. Reactive oxygen species play a central role in the activity of cationic liposome based cancer vaccine. J. Control. Release 130, 22-28 (2008).

61. Getts, D. R. et al. Therapeutic inflammatory monocyte modulation using immune-modifying microparticles. Sci. Transl. Med. 6, 219 ra217 (2014).

62. Zupancic, E. et al. Rational design of nanoparticles towards targeting antigen-presenting cells and improved T cell priming. J. Control. Release 258, 182-195 (2017)

63. Bachmann, M. F. et al. The influence of antigen organization on B cell responsiveness. Science 262, 1448-1451 (1993).

64. Cruz, L. J., Tacken, P. J., Fokkink, R. \& Figdor, C. G. The influence of PEG chain length and targeting moiety on antibody-mediated delivery of nanoparticle vaccines to human dendritic cells. Biomaterials 32 6791-6803 (2011).

65. Landgraf, M. et al. Targeted camptothecin delivery via silicon nanoparticles reduces breast cancer metastasis. Biomaterials 240, 119791 (2020).

66. Yong, K. W., Yuen, D., Chen, M. Z., Porter, C. J. H. \& Johnston, A. P. R. Pointing in the right direction: controlling the orientation of proteins on nanoparticles improves targeting efficiency. Nano Letters 19, 1827-1831 (2019).

67. Eisenbarth, S. C. Dendritic cell subsets in T cell programming: location dictates function. Nat. Rev. Immunol. 19, 89-103 (2019).

68. Segura, E. Review of mouse and human dendritic cell subsets. Methods Mol. Biol. 1423, 3-15 (2016)

69. Mintern, J. D. et al. Targeting dendritic cells: the role of specific receptors in the internalization of polymer capsules. Adv. Healthc. Mater. 2, 940-944 (2013).

70. Joffre, O. P., Sancho, D., Zelenay, S. \& Keller, A. M. \& Reis e Sousa, C Efficient and versatile manipulation of the peripheral CD4+ T-cell compartment by antigen targeting to DNGR-1/CLEC9A. Eur. J. Immunol. 40, 1255-1265 (2010).

71. Caminschi, I. et al. The dendritic cell subtype-restricted C-type lectin Clec9A is a target for vaccine enhancement. Blood 112, 3264-3273 (2008).

72. Petzold, C., Schallenberg, S., Stern, J. N. H. \& Kretschmer, K. Targeted antigen delivery to DEC- $205^{+}$dendritic cells for tolerogenic vaccination. Rev. Diabet. Stud. 9, 305-318 (2012).

73. Schetters, S. T. T. et al. Mouse DC-SIGN/CD209a as target for antigen delivery and adaptive immunity. Front. Immunol. https://doi.org/10.3389/ fimmu.2018.00990 (2018).

74. Guilliams, M. et al. Dendritic cells, monocytes and macrophages: a unified nomenclature based on ontogeny. Nat. Rev. Immunol. 14, 571-578 (2014).

75. Hlavaty, K. A., Luo, X., Shea, L. D. \& Miller, S. D. Cellular and molecular targeting for nanotherapeutics in transplantation tolerance. Clin. Immunol. 160, 14-23 (2015).

76. Kishimoto, T. K. et al. Improving the efficacy and safety of biologic drugs with tolerogenic nanoparticles. Nat. Nanotechnol. 11, 890-899 (2016)

77. Zheng, $\mathrm{X}$. et al. Treatment of autoimmune arthritis using RNA interference-modulated dendritic cells. J. Immunol. 184, 6457 (2010).

78. Yeste, A., Nadeau, M., Burns, E. J., Weiner, H. L. \& Quintana, F. J. Nanoparticle-mediated codelivery of myelin antigen and a tolerogenic small molecule suppresses experimental autoimmune encephalomyelitis. Proc. Natl Acad. Sci. USA 109, 11270-11275 (2012).

79. Metcalfe, S. M. \& Fahmy, T. M. Targeted nanotherapy for induction of therapeutic immune responses. Trends Mol. Med. 18, 72-80 (2012).

80. Wood, K. J., Shankar, S., Hester, J. \& Issa, F. Clinical Immunology 5th Edition (Elsevier, 2019)

81. Page, E., Dar, W. \& Knechtle, S. Tolerogenic therapies in transplantation. Front. Immunol. https://doi.org/10.3389/fimmu.2012.00198 (2012).

82. Zhuang, Q. et al. Graft-infiltrating host dendritic cells play a key role in organ transplant rejection. Nat. Commun. 7, 12623 (2016).

83. Schroeder, R. A., Marroquin, C. E. \& Kuo, P. C. Tolerance and the "Holy Grail" of transplantation. J. Surg. Res. 111, 109-119 (2003).

84. Bryant, J. et al. Nanoparticle delivery of donor antigens for transplant tolerance in allogeneic islet transplantation. Biomaterials 35 8887-8894 (2014).

85. Hlavaty, K. A. et al. Tolerance induction using nanoparticles bearing HY peptides in bone marrow transplantation. Biomaterials 76, 1-10 (2016).

86. Price, J. D. \& Tarbell, K. V. The role of dendritic cell subsets and innate immunity in the pathogenesis of type 1 diabetes and other autoimmune diseases. Front. Immunol. 6, 288 (2015).

87. Bottino, R., Knoll, M. F., Knoll, C. A., Bertera, S. \& Trucco, M. M. The future of islet transplantation is now. Front. Med. 5, 202 (2018).
88. Kleijwegt, F. S. et al. Transfer of regulatory properties from tolerogenic to proinflammatory dendritic cells via induced autoreactive regulatory $\mathrm{T}$ cells. J. Immunol. 187, 6357-6364 (2011).

89. Volchenkov, R., Karlsen, M., Jonsson, R. \& Appel, S. Type 1 regulatory $\mathrm{T}$ cells and regulatory B cells induced by tolerogenic dendritic cells. Scand. J. Immunol. 77, 246-254 (2013).

90. Mukhopadhaya, A. et al. Selective delivery of beta cell antigen to dendritic cells in vivo leads to deletion and tolerance of autoreactive CD8+ T cells in NOD mice. Proc. Natl Acad. Sci. USA 105, 6374-6379 (2008).

91. Price, J. D., Hotta-Iwamura, C., Zhao, Y., Beauchamp, N. M. \& Tarbell, K. V. DCIR2+ cDC2 DCs and Zbtb32 restore CD4+ T-Cell tolerance and inhibit diabetes. Diabetes 64, 3521-3531 (2015).

92. Yeste, A. et al. Tolerogenic nanoparticles inhibit T cell-mediated autoimmunity through SOCS2. Sci. Signal. 9, ra61 (2016).

93. Xie, Z. X. et al. Role of the immunogenic and tolerogenic subsets of dendritic cells in multiple sclerosis. Mediators Inflamm. 2015, 513295 (2015)

94. Dos Passos, G. R., Sato, D. K., Becker, J. \& Fujihara, K. Th17 cells pathways in multiple sclerosis and neuromyelitis optica spectrum disorders: pathophysiological and therapeutic implications. Mediators Inflamm. 2016, 5314541 (2016).

95. Wang, L., Li, Z., Ciric, B., Safavi, F., Zhang, G. X. \& Rostami, A. Selective depletion of CD11c. Eur. J. Immunol. 46, 2454-2466 (2016).

96. Flórez-Grau, G., Zubizarreta, I., Cabezón, R., Villoslada, P. \& Benitez-Ribas, D. Tolerogenic dendritic cells as a promising antigen-specific therapy in the treatment of multiple sclerosis and neuromyelitis optica from preclinical to clinical trials. Front. Immunol. 9, 1169 (2018).

97. Cappellano, G. et al. Subcutaneous inverse vaccination with PLGA particles loaded with a MOG peptide and IL-10 decreases the severity of experimental autoimmune encephalomyelitis. Vaccine 32, 5681-5689 (2014).

98. Getts, D. R. et al. Microparticles bearing encephalitogenic peptides induce T-cell tolerance and ameliorate experimental autoimmune encephalomyelitis. Nat. Biotechnol. 30, 1217-1224 (2012).

99. Papenfuss, T. L. et al. Estriol generates tolerogenic dendritic cells in vivo that protect against autoimmunity. J. Immunol. 186, 3346-3355 (2011).

100. LaMothe, R. A. et al. Tolerogenic nanoparticles induce antigen-specific regulatory $\mathrm{T}$ cells and provide therapeutic efficacy and transferrable tolerance against experimental autoimmune encephalomyelitis. Front. Immunol. 9, 281 (2018).

101. Constantinescu, C. S., Farooqi, N., O’Brien, K. \& Gran, B. Experimental autoimmune encephalomyelitis (EAE) as a model for multiple sclerosis (MS). Br. J. Pharmacol. 164, 1079-1106 (2011)

102. Stern, J. N. et al. Promoting tolerance to proteolipid protein-induced experimental autoimmune encephalomyelitis through targeting dendritic cells. Proc. Natl Acad. Sci. USA 107, 17280-17285 (2010).

103. Tabansky, I. et al. Targeting DEC-205(-)DCIR2(+) dendritic cells promotes immunological tolerance in proteolipid protein-induced experimental autoimmune encephalomyelitis. Mol. Med. 24, 17 (2018).

104. Loschko, J. et al. Antigen targeting to plasmacytoid dendritic cells via Siglec-H inhibits Th cell-dependent autoimmunity. J. Immunol. 187, 6346-6356 (2011).

105. Wing, J. B., Tanaka, A. \& Sakaguchi, S. Human FOXP3(+) regulatory T Cell heterogeneity and function in autoimmunity and cancer. Immunity $\mathbf{5 0}$, 302-316 (2019).

\section{Acknowledgements}

A.C-R. is currently supported by a National Health \& Medical Research Council (NHMRC) of Australia fellowship (GNT1112432). A.P.R.J is supported by an NHMRC fellowship (GNT1141551) and Australian Research Council (DP200100475, CE140100036). This work was performed in part at the Melbourne Centre for Nanofabrication (MCN) in the Victorian Node of the Australian National Fabrication Facility (ANFF).

\section{Competing interests}

The authors declare no competing interests.

\section{Additional information \\ Correspondence should be addressed to A.C.-R. or N.H.V.}

Peer review information Nature Nanotechnology thanks the anonymous reviewers for their contribution to the peer review of this work.

Reprints and permissions information is available at www.nature.com/reprints.

Publisher's note Springer Nature remains neutral with regard to jurisdictional claims in published maps and institutional affiliations.

(c) Springer Nature Limited 2020 